\title{
The Calculation of Multidimensional Hermite Polynomials and Gram-Charlier Coefficients*
}

\author{
By S. Berkowitz and F. J. Garner
}

\begin{abstract}
The paper documents derivations of:
(a) a recurrence relation for calculating values of multidimensional Hermite polynomials,

(b) a recurrence relation for calculating an approximation to the Gram-Charlier coefficients of the probability density distribution associated with a random process, based on (a),

(c) an efficient algorithm to utilize the formulae in (a) and (b).
\end{abstract}

I. Introduction. The paper documents derivations of: (a) a recurrence relation for calculating values of multidimensional Hermite polynomials; (b) a recurrence relation for calculating an approximation to the Gram-Charlier coefficients of the probability density distribution associated with a random process, based on (a); (c) an efficient algorithm to utilize the formulae in (a) and (b).

The relations for producing Hermite polynomials are generalizations of formulae derived in Appell and Kampé de Fériet [1]. The recurrence relation mentioned in (a) above appears without derivation in Vilenkin [5], which further references a dissertation by Sirazhdinov [4].

Since Hermite polynomials form complete, biorthogonal systems with respect to the Gaussian probability density, one basic use of the polynomials is to expand a near-Gaussian probability density distribution in terms of the polynomials in a socalled Gram-Charlier series. As we shall demonstrate below, the coefficients of the series can be calculated directly from the time series generated by a random process.

Finally, we present and prove an algorithm which computes a Hermite polynomial or Gram-Charlier coefficient of vector order $\mathrm{m}$ by means of the above recurrence relations. The algorithm requires the smallest number of suborder polynomials and/or coefficients possible.

The derivation of the recurrence relations is the responsibility of S. Berkowitz, and the algorithm represents the work of both authors. The algorithm has been implemented in FORTRAN subroutines by Mr. Garner.

\section{Multidimensional Hermite Polynomials.}

A. Definitions.

1. $\mathbf{m}=\left(m_{1}, \cdots, m_{n}\right)$ is an order vector of degrees $m_{i}$. The polynomial or argument $\mathrm{x}$ and of index $\mathrm{m}$ is said to be of degree $m_{i}$ in the argument $x_{i}(i=1, \cdots, n)$ and of total degree $m=\sum_{i=1}^{n} m_{i}$.

Received August 22, 1969.

AMS Subject Classifications. Primary 3340; Secondary 6020, 6525.

Key Words and Phrases. Multidimensional Hermite polynomials, Gram-Charlier series, recurrence relations, Gaussian probability density, normal distributions.

* This paper was written in the Applied Mathematics Laboratory of the Naval Ship Research and Development Center for Project No. 1-830-918-02.

Copyright $\odot$ 1971, American Mathematical Society 
2. $\mathbf{x}=\left(x_{i}, \cdots, x_{n}\right)$ is a vector of arguments $x_{i}$.

3. $R \equiv Q^{-1}$ is a positive definite matrix. One calls $R$ a covariance matrix for the particular application of expanding joint probability density distributions in a GramCharlier series. $R \equiv\left[r_{i i}\right] ; Q \equiv\left[q_{i j}\right]$.

4. The complete biorthogonal systems of Hermite polynomials $\left\{\boldsymbol{H}_{\mathbf{m}}(\mathbf{x})\right\}$ and $\left\{G_{\mathrm{m}}(\mathrm{x})\right\}$ are defined by the generating functions:

$$
\begin{aligned}
& \exp \left[\mathrm{a}^{T} Q \mathrm{x}-\frac{1}{2} \mathrm{a}^{T} Q \mathrm{a}\right]=\sum_{m=0}^{\infty} c_{\mathrm{m}}(\mathrm{a}) H_{\mathrm{m}}(\mathrm{x}), \\
& \exp \left[\mathrm{a}^{T} \mathrm{x}-\frac{1}{2} \mathrm{a}^{T} R \mathrm{a}\right]=\sum_{m=0}^{\infty} c_{\mathrm{m}}(\mathrm{a}) G_{\mathrm{m}}(\mathrm{x}),
\end{aligned}
$$

where

(i) $c_{\mathrm{m}}$ (a) $=\coprod_{i=1}^{n} a_{i}^{m_{i}} / m_{i} !$,

(ii) the $a_{i}$ are arbitrary.

The sets $\left\{H_{\mathrm{m}}(\mathbf{x})\right\}$ and $\left\{G_{\mathbf{m}}(\mathbf{x})\right\}$ are biorthogonal with respect to $(\operatorname{det} Q)^{1 / 2}(2 \pi)^{-n / 2}$ $\cdot \exp \left[-\frac{1}{2} \mathbf{x}^{T} Q \mathbf{x}\right]$ over the entire real $n$-space. In the case of unidimensional polynomials $(n=1)$, both $\left\{H_{\mathrm{m}}(\mathbf{x})\right\}$ and $\left\{G_{\mathrm{m}}(\mathbf{x})\right\}$ reduce to a single orthogonal, complete set $\left\{H_{m}(x)\right\}$, which is generated by the following relation (cf. Erdélyi [2]):

$$
\exp \left[a x-\frac{1}{2} a^{2}\right]=\sum_{m=0}^{\infty} \frac{a^{m}}{m !} H_{m}(x) .
$$

The polynomials $\left\{H_{m}(x)\right\}$ are orthogonal with respect to exp $\left[-\frac{1}{2} x^{2}\right]$ over the entire real line.

B. Recurrence Relations for $H_{\mathrm{m}}(\mathbf{x})$ and $G_{\mathrm{m}}(\mathbf{x})$. Differentiating the generating functions (1) with respect to $a_{k}$ produces

$$
\begin{aligned}
\frac{\partial}{\partial a_{k}}\left\{\exp \left[\mathbf{a}^{T} Q \mathbf{x}-\mathbf{a}^{T} Q \mathbf{a}\right]\right\} & =\sum_{j=1}^{n} q_{k j}\left(x_{j}-a_{j}\right) \sum_{m=0}^{\infty} c_{\mathrm{m}}(\mathbf{a}) H_{\mathrm{m}}(\mathbf{x}) \\
& =\sum_{m=0}^{\infty}\left(\prod_{i \neq k} \frac{a_{i}^{m_{i}}}{m_{i} !}\right) \frac{a_{k}^{m_{k}-1}}{\left(m_{k}-1\right) !} H_{\mathrm{m}}(\mathbf{x}) .
\end{aligned}
$$

By matching coefficients of $a_{k}^{m_{k}-1} \coprod_{i \neq k} a_{i}^{m_{i}}$ in the right-hand equation of (4) one finds (5)

$$
\begin{array}{r}
H_{\mathrm{m}}(\mathbf{x})=\left(\sum_{j=1}^{n} q_{k j} x_{i}\right) H_{\mathrm{m}-\mathbf{e}_{k}}(\mathbf{x})-\sum_{j \neq k} q_{k j} m_{j} H_{\mathbf{m}-\mathbf{e}_{k-e_{j}}}(\mathbf{x})-q_{k k}\left(m_{k}-1\right) H_{\mathbf{m}-\mathbf{e}_{k}}(\mathbf{x}), \\
k=1, \cdots, n,
\end{array}
$$

where $\mathbf{e}_{k}$ is a vector with a " 1 " as the $k$ th component, and zeroes elsewhere. A similar operation on the generating function (2) produces:

$$
\begin{array}{r}
G_{\mathbf{m}}(\mathbf{x})=x_{k} G_{\mathbf{m}-\mathbf{e}_{k}}(\mathbf{x})-\sum_{j \neq k} r_{k j} m_{j} G_{\mathbf{m}-\mathbf{e}_{k-\mathbf{e}_{j}}(\mathbf{x})-\left(m_{k}-1\right) r_{k k} G_{\mathbf{m}-2 \mathbf{e} k}(\mathbf{x}),} \\
k=1, \cdots, n .
\end{array}
$$

For any particular $H_{\mathrm{m}}(\mathbf{x})$ or $G_{\mathrm{m}}(\mathbf{x})$, one has a choice of $n$ recurrence relations. The value of any term in (5) or (6) with a negative index is zero and the $j$ th relation must not be used if $m_{i}=0$ as one would falsely conclude $H_{\mathrm{m}}(\mathrm{x})=0$. If one chooses the $p$ th recurrence relation to compute $H_{\mathrm{m}}(\mathbf{x})$ or $G_{\mathrm{m}}(\mathbf{x})$, then $m_{p}$ is called the pivot order, and $p$ is called the pivot. 
III. Calculation of Gram-Charlier Series Coefficients. Consider the $n$-dimensional vector time series $\mathbf{x}^{(1)}, \mathbf{x}^{(2)}, \ldots$ generated from a random process whose joint probability density is $p(\mathbf{x})$.

The components of the $k$ th vector of the time series are:

$$
\mathbf{x}^{(k)}=x_{1}^{(k)}, \cdots, x_{n}^{(k)} .
$$

Denote the Gaussian probability density by the formula:

$$
p_{0}(\mathbf{x})=(\operatorname{det} Q)^{1 / 2}(2 \pi)^{-n / 2} \exp \left[-\frac{1}{2}(\mathbf{x}-\boldsymbol{u})^{T} Q(\mathbf{x}-\boldsymbol{u})\right],
$$

where (a) $\boldsymbol{u}=\mu_{1}, \cdots, \mu_{n}$ is usually taken as the vector of ensemble averages of the variates $x_{1}, \cdots, x_{n}$, respectively.

(b) $Q$ is a positive definite $n$ by $n$ matrix, usually taken as the inverse covariance matrix of $x$.

One can expand $p(\mathbf{x})$ in a Gram-Charlier series as follows:

$$
p(\mathbf{x})=p_{0}(\mathbf{x}) \sum_{m=1}^{\infty} A_{\mathrm{m}} H_{\mathrm{m}}(\mathrm{y}),
$$

where (a) $\mathrm{y}=y_{1}, \cdots, y_{n}$ is a normalization of $\mathbf{x}$ such that $y_{i}=\left(x_{i}-\mu_{i}\right) \sigma_{i}$, $(i=1, \cdots, n)$, and

(b) $\sigma_{i}=E\left[\left(x_{i}-\mu_{i}\right)^{2}\right]$ is the second moment of $x_{i}$ about its expectation.

The biorthogonality property of the $H_{\mathrm{m}}(\mathbf{x})$ and $G_{\mathrm{m}}(\mathbf{x})$ is:

$$
\int_{S} p_{0}(\mathbf{x}) G_{\mathrm{m}}(\mathrm{x}) H_{\mathrm{p}}(\mathrm{x}) d \mathrm{x}=b_{\mathrm{m}}^{-1} \prod_{i=1}^{n} \delta_{m_{i} p_{i}}
$$

where: (a) $S$ indicates the entire real $n$-space,

(b) $b_{\mathrm{m}}=\prod_{i=1}^{n}\left(m_{i} !\right)^{-1}$,

(c) $\delta_{m p}$ is the Kronecker delta.

By means of the biorthogonality property (8) and the Gaussian weighting function $p_{0}(\mathbf{x})$, one can formulate the Gram-Charlier coefficients as follows:

$$
A_{\mathrm{m}}=b_{\mathrm{m}} \int_{S} G_{\mathrm{m}}(\mathrm{y}) p(\mathbf{x}) d \mathbf{x},
$$

or, simply,

$$
A_{\mathrm{m}}=b_{\mathrm{m}} E\left[G_{\mathrm{m}}(\mathrm{y})\right] .
$$

From (9) and the recurrence relation (6), one deduces:

$$
A_{\mathrm{m}}=b_{\mathrm{m}}\left[E\left[y_{k} G_{\mathrm{m}-e_{k}}(\mathrm{y})\right]-\sum_{j=1}^{n} r_{k j} m_{j} E\left[G_{\mathrm{m}-\mathrm{e}_{k-e_{j}}}(\mathrm{y})\right]+r_{k k} E\left[G_{\mathrm{m}-2 e_{k}}(\mathrm{y})\right]\right] .
$$

For a sample set of $M$ vectors of the type indicated by (7), the first term on the right-hand side of (10) may be written as

$$
E\left[y_{k} G_{\mathrm{m}-\mathrm{e}_{k}}(\mathrm{y})\right] \approx \frac{1}{M} \sum_{i=1}^{M} y_{k}^{(i)} G_{\mathrm{m}-\mathrm{e}_{k}}\left(\mathrm{y}^{(i)}\right) .
$$


The expectations $\mu_{k}$ and $\sigma_{k}$ have approximations

$$
\begin{aligned}
& \mu_{k} \approx \frac{1}{M} \sum_{i=1}^{M} x_{k}^{(i)}, \\
& \sigma_{k} \approx \frac{1}{M} \sum_{i=1}^{M}\left(x_{k}^{(i)}-\mu_{k}\right)^{2} .
\end{aligned}
$$

Thus, by substituting (11) and (9) into (10) one has a recurrence relation for $A_{\mathrm{m}}$ (which, in turn, employs the recurrence relation (6) for $G_{\mathbf{m}}(\mathbf{x})$ ):

$$
A_{\mathrm{m}}=\frac{1}{M}\left[b_{\mathrm{m}} \sum_{i=1}^{M} y_{k}^{(i)} G_{\mathrm{m}-\mathrm{e}_{k}}\left(\mathrm{~g}^{(i)}\right)-\sum_{j=1}^{n} r_{k j} m_{k}^{-1} A_{\mathrm{m}-\mathrm{e}_{k}-\mathrm{e}_{j}}\right], \quad k=1, \cdots, n .
$$

Similarly, for the expansion

$$
p(\mathbf{x})=p_{0}(\mathbf{x}) \sum_{m=0}^{\infty} B_{\mathrm{m}} G_{\mathrm{m}}(\mathrm{y}),
$$

one can derive the recurrence relation:

$$
\begin{array}{r}
B_{\mathrm{m}}=\frac{1}{M}\left[b_{\mathrm{m}} \sum_{i=1}^{M} \sum_{i=1}^{n} q_{k i} y_{i}^{(i)} H_{\mathrm{m}-\mathrm{ek}}\left(\mathrm{y}^{(i)}\right)-\sum_{i=1}^{n} q_{k i} m_{k}^{-1} B_{\mathrm{m}-\mathrm{ek}-\mathrm{e} i}\right], \\
k=1, \cdots, n .
\end{array}
$$

As in the recurrence relations for Hermite polynomials, any term in (14), or (15) with a negative index is to be regarded as zero. In fact, since the indexing in (14) and (15) corresponds (with a bit of manipulation) to the indexing in (5) and (6), the problems of sequencing the recursive generation of Gram-Charlier coefficients are the same as those for sequencing Hermite polynomials, with the exception that only the vectors $\mathrm{m}-\mathbf{e}_{k}-\mathbf{e}_{i}, j=1, \cdots, n$, need be generated for the Gram-Charlier coefficients. Therefore, in the remainder of the paper, we restrict ourselves to a discussion of Hermite polynomials and make only parenthetical restrictions where necessary for Gram-Charlier coefficients.

IV. An Algorithm for Generating Values of Hermite Polynomials (or GramCharlier Coefficients). In recursively generating the value of $H_{\mathrm{m}}(\mathbf{x})$ or $G_{\mathrm{m}}(\mathbf{x})$ from (5) or (6) one could conceivably generate all $H_{\mathrm{v}}(\mathrm{x})$ or $G_{\mathrm{v}}(\mathrm{x})$ such that $v_{i} \leqq m_{i}(i=1$, $\cdots, n)$. However, there is a test that permits one to determine, purely on the basis of the order vector $v$, whether or not a polynomial value need be calculated from the recurrence relation. The test is embodied in an algorithm which we present below and prove in the next section. The algorithm requires a prior decision on the generating sequence as follows:

The order vector $\mathrm{m}$ is permuted according to an arbitrary but fixed pivot sequence vector

$$
\boldsymbol{\delta}=\sigma(1), \cdots, \sigma(n),
$$

where the set $\{\sigma(j)\}$ is a permutation of $1, \cdots, n$. The classes $V_{p}$, defined by 


$$
\begin{aligned}
V_{p}=\left\{\nabla=v_{1}, \cdots, v_{n} \mid 0 \leqq v_{\sigma(i)} \leqq m_{\sigma(j)}, 1 \leqq j \leqq p-1 ;\right. \\
\left.0<v_{\sigma(p)} \leqq m_{\sigma(p)} ; v_{\sigma(j)}=0, p+1 \leqq j \leqq n\right\}, \\
\quad p=1, \cdots, n,
\end{aligned}
$$

are each generated according to an ordering $S_{p}$ which is defined by

$$
\begin{aligned}
S_{p}=\left\{v, v^{\prime} \in V_{p} \mid\right. & v=\left(v_{\sigma(1)}, \cdots, v_{\sigma(p)}, 0, \cdots, 0\right), \\
\mathbf{v}^{\prime} & =\left(v_{\sigma(1)}^{\prime}, \cdots, v_{\sigma(p)}^{\prime}, 0, \cdots, 0\right) \text {, and } \\
& \left.<<v^{\prime} \text { iff } \sum_{i=1}^{p}\left(v_{\sigma(j)}-v_{\sigma(i)}^{\prime}\right) b^{p-i}<0, \text { where } b>\max _{i}\left(m_{i}\right)\right\} .
\end{aligned}
$$

The class $V_{p}$ stipulates that the $\sigma(p)$ th recurrence relation is to be used for calculating $H_{\mathrm{v}}(\mathrm{x})$ if $\mathrm{v} \in V_{p}$. The sequence $S_{p}$ is merely an ordering of the real numbers $\left\{\left(v_{\sigma(1)} v_{\sigma(2)} \cdots v_{\sigma(p)} b\right\}\right.$ in a base $b$ sufficiently large so that no $v_{\sigma(j)}$ exceeds $b-1$, thus insuring the availability of lower-ordered polynomial values for the calculation of a given polynomial value in the recurrence relation.

The algorithm now follows:

1. Set $H_{0}(\mathrm{x})=1\left(\right.$ or $\left.G_{0}(\mathrm{x}) \equiv A_{0} \equiv B_{0} \equiv 1\right)$.

2. Initialize the pivot sequence at $p=1$.

3. Initialize the order vector sequence in $S_{p}$.

4. For the ordering $S_{p}$ over $V_{p}$, generate the sth order vector $\mathbf{v}$.

5. (a) If

$$
\sum_{i=p+1}^{n} m_{\sigma(i)}+\left(m_{\sigma(p)}-v_{\sigma(p)}\right) \geqq \sum_{j=1}^{p-1}\left(m_{\sigma(i)}-v_{\sigma(i)}\right),
$$

compute $H_{\mathrm{v}}(\mathrm{x})$ (or $G_{\mathrm{v}}(\mathrm{x})$ ) from the $\sigma(p)$ th recurrence relation and store. (For the computation of a Gram-Charlier coefficient, we require also that $\sum_{i=1}^{n}\left(m_{i}-v_{i}\right)$ be even.)

(b) Otherwise, proceed directly to 6.

6. (a) If $V_{p}$ is not exhausted, update $s$ by one and return to 4 to increment $V_{p}$ according to $S_{p}$.

(b) If $V_{p}$ is exhausted, and $p<n$, update $p$ by one and return to 3 to initialize $S_{p}$.

(c) If $p=n$, exit.

The number of polynomial values required to compute the value of a given $H_{\mathrm{m}}(\mathrm{x})$ or $G_{\mathrm{m}}(\mathrm{x})$ depends heavily upon the manner in which one chooses the order sequence. The order sequence (16) which minimizes the number of required polynomial values is

$$
j \leqq k \quad \text { iff } \quad m_{\sigma(i)} \geqq m_{\sigma(k)} .
$$

We will justify this assertion after presenting the proof for the algorithm.

V. Proof of the Algorithm.

A. Definitions. The reverse sequence of indices in the following definitions facilitates the description of index increments at the top of the partial ordering of order vectors required by the recurrence relation. 
1. Order Vectors.

(a) Target Order Vector $\mathrm{h} \equiv h_{n}, \cdots, h_{1}, \equiv m_{\sigma(1)}, \cdots, m_{\sigma(n)}$.

(b) Test Order Vector $\mathrm{w} \equiv w_{n}, \cdots, w_{1}, \equiv v_{\sigma(1)}, \cdots, v_{\sigma(n)}$.

(c) Test Difference $d_{i} \equiv h_{j}-w_{i}, j=1, \cdots, n$.

(d) Masking Vector $\mathrm{e}^{\prime}{ }_{i} \equiv \mathrm{e}_{n-j+1} \equiv\left(\delta_{n i}, \cdots, \delta_{1 i}\right), j=1, \cdots, n$.

2. Pivot Classes.

(a) $C_{p} \equiv V_{n-p+1}, p=1, \cdots, n$. (Note that the $C_{p}$ form a partition of the set of suborder vectors to be considered in computing $\mathbf{h}$.)

(b) $T_{p}=\left\{\mathrm{w} \mid \mathrm{w} \in C_{p}\right.$ and $\left.\sum_{i=1}^{p} d_{i} \geqq \sum_{i-p+1}^{n} d_{i}, p=1, \cdots, n\right\}$.

(c) $R=\left\{\mathrm{w} \mid \mathrm{w}\right.$ is needed in a recurrence relation to compute $H_{\mathrm{m}}(\mathbf{x})$ or $\left.G_{\mathrm{m}}(\mathbf{x})\right\}$.

(d) The pivot associated with $C_{p}$ or $T_{p}$ is $\sigma(n-p+1)$; that is, if w $\in T_{p} \cap R$, then the $\sigma(n-p+1)$ th recurrence relation is used to compute $H_{\mathrm{v}}(\mathbf{x})$ or $G_{\mathrm{v}}(\mathbf{x})$.

3. Increment Classes.

(a) According to the recurrence relation (5) or (6), there are three types of increments:

Type I-The pivot order $w_{p}$ is incremented by one.

Type II-The pivot order $w_{p}$ is incremented by two. (This type is equivalent to two Type I increments, and will not be used below.)

Type III-The pivot order $w_{p}$ is incremented by one, and some other order $w_{i}$ $(j>p)$ is also incremented by one.

(b) A vector a $=a_{n}, \cdots, a_{1}$ is in $L_{p}(t, \mathrm{w})$ if and only if a is the result of zero or more increments from w with pivot $\sigma(n-p+1)$ and increment Type(s) $t=\mathrm{I}$, II, or III.

B. The Proof. The following two notes labelled (N1) and (N2) will be employed in the proof.

When a pivot remains constant throughout incrementing from $w$ to a,

(N1) the recurrence relations demand that $a_{p}-w_{p} \geqq \sum_{i \neq p}\left(a_{i}-w_{i}\right)$, where the pivot is $\sigma(n-p+1)$.

(N2) If any chain of increments can be constructed from $\mathrm{w}$ to $\mathrm{h}$, then $\mathrm{w} \in R$.

We seek to prove that $R=\bigcup_{k} T_{k}$. However, since the $C_{k}$ form a partition of the order vectors under consideration, $R=\bigcup_{k}\left(R \cap C_{k}\right)$, it suffices to prove that

$$
R \cap C_{k}=T_{k}, \quad k=1, \cdots, n,
$$

by induction on $k$.

1. For $k=1$.

(a) Assume $\mathrm{w} \in R \cap C_{1}$. The pivot is $\sigma(n)$, since $\mathrm{w} \in C_{1}$. Since, by (N1), $d_{1} \geqq$ $\sum_{i=2}^{n} d_{j}$, it is true that $\mathrm{w} \in T_{1}$ and thus $T_{1} \subset R \cap C_{1}$.

(b) Assume w $\in T_{1}$. Thus, $d_{1} \geqq \sum_{i=2}^{n} d_{i}$.

(i) If the right-hand side of the inequality is zero, then $\mathrm{h} \in L_{1}(\mathrm{I}, \mathrm{w})$ and, by (N2), w $\in R$. Since, $T_{1} \subset C_{1}$, w $\in R \cap C_{1}$.

(ii) If the right-hand side of the inequality is positive, then $\exists w_{1}=\mathrm{h}-$ $\left(d_{1}-\sum_{i=2}^{n} d_{i}\right) \mathrm{e}_{1}^{\prime} \in L_{1}(\mathrm{III}, \mathrm{w})$. But $\mathrm{h} \in L_{1}\left(\mathrm{I}, \mathrm{w}_{1}\right)$ and, by (N2), $\mathrm{w} \in R$. As in (i) then, $T_{1} \subset R \cap C_{1}$.

$$
\therefore T_{1}=R \cap C_{1} \text {. }
$$

2. Suppose $T_{k}=R \cap C_{k}, 1 \leqq k \leqq m-1<n$. 
We show now that $T_{m}=R \cap C_{m}$.

(a) Assume $\mathrm{w} \in R \cap C_{m}$. There are three cases:

(i) $\mathrm{w}=\mathrm{h}$;

(ii) $\exists \mathrm{a}=\left(a_{n}, \cdots, a_{p+1}, w_{p}+1,0, \cdots, 0\right) \in L_{p}$ (I or III, w), for $p<m$. By definition, $a \in C_{p}$. Moreover, if a is also in $R$, then, by ( $\left.\mathrm{H} 1\right), a \in T_{p}$. If one defines $d_{i}^{\prime}=h_{i}-a_{i}, j=1, \cdots, n$, then one has

$$
\begin{aligned}
\sum_{i=1}^{m} d_{i} & \geqq \sum_{i=1}^{p} d_{i}=\sum_{i=1}^{p} d_{i}^{\prime}+1 \\
& \geqq \sum_{i=p+1}^{n} d_{i}^{\prime}+\sum_{i=p+1}^{n}\left(a_{i}-w_{i}\right) \quad \text { (by (H1) and (N1)) } \\
& \geqq \sum_{i=m+1}^{n} d_{i} .
\end{aligned}
$$

Thus, $\mathrm{w} \in T_{m}$ if any a of the above type is in $R$;

(iii) $\exists \mathrm{b}=\left(b_{n}, \cdots, b_{m+1}, h_{m}, 0, \cdots, 0\right) \in L_{m}$ (I and/or II and/or III, w). By definition, $\mathrm{b} \in C_{m}$. Consider any $\mathrm{c}=\left(c_{n}, \cdots, c_{p+1}, 1,0, \cdots, 0\right) \in L_{p}(\mathrm{I}$ or III, b) for $p<m$. If $\mathrm{c} \in R$, then, as in (ii) above, $\mathrm{b} \in T_{m}$. Let $d_{i}^{\prime}=h_{j}-b_{i}, j=1, \cdots, n$. The following inequalities show that $\mathrm{w} \in T_{m}$ (if $\mathrm{c} \in R$ ):

$$
\begin{aligned}
\sum_{i=1}^{m} d_{i} & \geqq \sum_{i=1}^{m-1} d_{i}^{\prime}+\sum_{i=m+1}^{n}\left(b_{i}-w_{i}\right) \quad(\text { by }(\mathrm{N} 1)) \\
& \geqq \sum_{m+1}^{n} d_{i}^{\prime}+\sum_{i=m+1}^{n}\left(b_{i}-w_{i}\right) \quad\left(\text { since } \mathrm{b} \in T_{m}\right) \\
& =\sum_{i=m+1}^{n} d_{i} .
\end{aligned}
$$

But increments of $\mathrm{b}$ for pivot $\sigma(n-p+1)$ with $p<m$ constitute all the increments of b. Moreover, any increment chain upward from $w$ produces at least one of the vectors represented by $h, a$, or $b$. Hence, at least one of the vectors must be in $R$. Therefore, $\mathrm{w} \in T_{m}$ and $R \cap C_{m} \subset T_{m}$.

(b) Assume $\mathrm{w} \in T_{m}$. We show that $\mathrm{w} \in R \cap C_{m}$ by induction on $d_{m}$.

(i) $d_{m}=0$, so that

$$
\sum_{i=1}^{m-1} d_{i} \geqq \sum_{j=m+1}^{n} d_{i} .
$$

Case One. If the right-hand side of (18) is zero, then either $\mathrm{w}=\mathrm{h}$ (in which case $\left.\mathrm{w} \in R \cap C_{1}\right)$, or $\exists p=\max \left\{j \mid h_{i}>0,1 \leqq j<m\right\}$, and $\exists \mathrm{c}=\mathrm{w}+\mathrm{e}_{p}^{\prime} \in L_{p}(\mathrm{I}, \mathrm{w})$. But c $\in T_{p}$, since $\sum_{j=1}^{p} h_{j} \geqq 1$. Hence, by (H1), c $\in R \cap C_{p}$, and, by (N2), w $\in R$. Since $T_{m} \subset C_{m}$, w $\in R \cap C_{m}$.

Case Two. On the other hand, if the right-hand side of the inequality (18) is positive, $\mathrm{w} \neq \mathrm{h}$ and $\exists k, m+1 \leqq k \leqq n$, such that $d_{k}>0$. Thus, for $p$ chosen as in Case One $-p$ exists, since otherwise $\mathrm{w}=\mathrm{h}-$ there is a vector $\mathrm{g}=\mathrm{g}_{n}, \cdots, \mathrm{g}_{1}=$ 
$\mathbf{w}+\mathbf{e}_{k}^{\prime}+\mathbf{e}_{p}^{\prime} \in L_{p}\left(\right.$ III, w). If one defines $d_{i}^{\prime} \equiv g_{i}-w_{i}, 1 \leqq j \leqq n$, then

$$
\sum_{i=1}^{p} d_{i}^{\prime}=\sum_{i=1}^{m-1} d_{i}-1 \geqq \sum_{i=m}^{n} d_{i}-1=\sum_{i=p+1}^{n} d_{i}^{\prime} .
$$

Thus, as in Case One, w $\in R \cap C_{m}$. Therefore, for $d_{m}=0, T_{m} \subset R \cap C_{m}$.

(ii) Suppose that $T_{m} \subset R \cap C_{m}$ for $0 \leqq d_{m}<t$.

We show now that if $\mathrm{w} \in T_{m}$ and if $d_{m}=t$, so that

$$
\sum_{i=1}^{m-1} d_{i}+t \geqq \sum_{i=m+1}^{n} d_{i},
$$

then $\mathrm{w} \in R \cap C_{m}$.

Case One. If the inequality in (19) is strict, then $\exists \mathrm{y}=y_{n}, \cdots, y_{1}=\mathbf{w}+$ $\mathrm{e}_{m}^{\prime} \in L_{m}(\mathrm{I}, \mathrm{w})$. If $d_{i}^{\prime} \equiv h_{i}-y_{i}, 1 \leqq j \leqq n$, then

$$
\sum_{i=1}^{m} d_{i}^{\prime}=\sum_{i=1}^{m-1} d_{i}+(t-1) \geqq \sum_{i=m+1}^{n} d_{i}=\sum_{i=m+1}^{n} d_{i}^{\prime} .
$$

Thus y $\in T_{m}$, and, since $d_{m}^{\prime}=t-1, \mathrm{y} \in R \cap C_{m}$ by (H2). As in Case One of (i) supra, we conclude that $\mathrm{w} \in R \cap C_{m}$.

Case Two. If, in (19), the inequality is in fact an equality, then $\exists k, m+1 \leqq k \leqq n$, such that $d_{k}>0$, and $\exists \mathrm{z}=z_{n}, \cdots, z_{1}=\mathrm{w}+\mathrm{e}_{m}^{\prime}+\mathrm{e}_{k}^{\prime} \in L_{m}$ (III, w). If $d_{i}^{\prime} \equiv$ $h_{i}-z_{i}, 1 \leqq j \leqq n$, then

$$
\sum_{i=1}^{m} d_{i}^{\prime}=\sum_{i=1}^{m-1} d_{i}+(t-1)=\sum_{i=m+1}^{n} d_{i}-1=\sum_{i=m+1}^{n} d_{i}^{\prime} .
$$

Thus, $\mathrm{z} \in T_{m}$, and, exactly as in Case One of (ii) $\mathrm{w} \in R \cap C_{m}$.

Therefore, $T_{m} \subset R \cap C_{m}$, and, indeed, $T_{m}=R \cap C_{m}$.

In applying the algorithm to Gram-Charlier coefficients, it was necessary that $\sum_{i=1}^{n}\left(m_{i}-v_{i}\right)$ be even. Let $\mathrm{u}$ be a type $t$ decrement of $\mathrm{w}$ if, and only if, $\mathrm{w}$ is a type $t$ increment of $\mathfrak{u}$. Then, in the recurrence relation (14) or (15), decrements of types II or III were needed to designate required order vectors for the $A_{\mathrm{m}}$ or $B_{\mathrm{m}}$. Hence for any required order vector, $\sum_{i=1}^{n} d_{i}$ must be even. Q.E.D.

Let us now justify the assertion that the order sequence (16) which minimizes the number of order vectors in $R$ is

$$
j \leqq k \quad \text { iff } m_{\sigma(i)} \geqq m_{\sigma(k)} \quad \text { (cf. (17)). }
$$

According to the algorithm, the vectors $w \in R \cap C_{p}$ are those specified by

$$
w_{p}-\sum_{i=p+1}^{n} w_{i} \leqq k_{p}, \quad p=1, \cdots, n,
$$

where

$$
k_{p}=\sum_{i=1}^{p} h_{i}-\sum_{i=p+1}^{n} h_{i}, \quad h_{i} \geqq w_{i} \geqq 0, \quad j=1, \cdots, n .
$$

Consider a Euclidean $(n-p+1)$-space $E_{p}$ with orthogonal axes $w_{p}, \cdots, w_{n}$. If one replaces the inequality in (20) by an equality, one has a hyperplane $R_{p}$ of (positive) unity slope with $w_{p}$-intersect at $w_{p}=k_{p}$. The points in $R \cap C_{p}$ form a closed subset 
of $E_{p}$, bounded by the hyperplanes $R_{p}, w_{i}=0$, and $w_{i}=h_{i}(i=p, \cdots, n)$, where for any $i, w_{j} \geqq 0$ if $j \neq i$. The number of points in $R \cap C_{p}$ is minimum for least $w_{p}$-intersect $k_{p}$, and thus for the minimum $\sum_{i=1}^{p} h_{i}$, a fact that justifies the assertion (17).

Naval Ship Research and Development Center

Washington, D. C. 20034

1. P. APPEll \& J. KAMPÉ DE FÉRIET, Fonctions Hypergéométriques et Hypersphériques: Polynômes d'Hermite, Gauthier-Villars, Paris, 1926.

2. A. ERDÉLYI, ET AL., Higher Transcendental Functions, McGraw-Hill, New York, 1953. MR 15, 419.

3. J. KAMPÉ DE FÉRIET, The Gram-Charlier Approximation of the Normal Law and the Statistical Description of a Homogeneous Turbulent Flow near Statistical Equilibrium, Applied Mathematics Laboratory, NSRDC Report No. 2013, March, 1966.

4. S. KH. SIRAzhdinov, Contribution to the Theory of Hermite Multidimensional Polynomials, Candidate Dissertation, Tashkent State University, USSR, 1949. (Russian)

5. S. YA. VILENKIN, "Determination of the maximum time (critical path time) distribution," Avtomat. $i$ Telemeh., v. 1965, no. 7, pp. 1247-1252 = Automat. Remote Control, v. 1965 , no. 7, pp. 1333-1337. 